\title{
Development of a Facile Method to Rebalance Oral Environment: Oral pH 7 Rebalancer
}

\author{
Brian Chung ${ }^{1}$, Jong Hyun $\mathrm{Im}^{1}$, Ji Young $\mathrm{Kim}^{1}$, Wonho $\mathrm{Ha}^{2}$ \\ ${ }^{1}$ Global Oral Health Lab, LG H\&H Research Center, Seoul, Korea, ${ }^{2}$ Oral Health R\&D Division, LG H\&H Research Center, \\ Seoul, Korea
}

Objective: We investigated whether a new chew-swish-swallow mouth refreshener, LG pH 7 Rebalancer tablet could control a drop in salivary $\mathrm{pH}$ and therefore improve the $\mathrm{pH}$ rebalancing potential of saliva after an acidic challenge such as a glucose rinse and high sugar foods by in-vivo and in-vitro studies.

Methods: Neutralized salivary $\mathrm{pH}$ after an acidic challenge was studied by in-vivo and in-vitro. For in-vivo study, participants were recruited for two visits, every morning for two days. At day 1, the baseline salivary $\mathrm{pH}$ of participants was measured and then rinsed with a $10 \%$ glucose solution for one minute as an acidic challenge. Their salivary $\mathrm{pH}$ was monitored at several time points for 60 minutes. At day 2, the same participants rinsed with a $10 \%$ glucose solution for one minute after checking the baseline $\mathrm{pH}$ of their saliva. Participants then were instructed to use a tablet (LG pH 7 rebalance) for 10 sec chewing and for 30 sec swishing and swallowed. The $\mathrm{pH}$ of saliva was measured immediately and at the same time points to 60 minutes. The $\mathrm{pH}$ rebalancing ability of the tablet was evaluated by comparing the two day studies. For in-vitro study, acid creation model was used: Streptococcus mutans (S. mutans) and high sugar containing food were dissolved in artificial saliva. The $\mathrm{pH}$ change upon the application of the tablet was measured at minute's interval to an overnight time periods.

Results: In all participants, the one minute rinse with 10\% glucose solution resulted in a period of acidic $\mathrm{pH}$, and the use of LG pH 7 rebalance tablet neutralized and substantially reduced the period time the $\mathrm{pH}$ maintained lower than 6.0. The average time period that the salivary $\mathrm{pH}$ was maintained acidic $\mathrm{pH}$ after the $10 \%$ glucose rinse was longer than 5 minutes. When the tablet was also used, the time for elevating $\mathrm{pH}$ was less than one minute. The in-vitro study also showed similar results: high sugar containing foods lowered the artificial saliva $\mathrm{pH}$ with $S$. mutans and rapid recovery of $\mathrm{pH}$ after taking the tablet was observed.

Conclusion: The use of a newly developed LG pH 7 Rebalancer tablet was shown drastic increase of the pH to control the lowering of salivary $\mathrm{pH}$ following carbohydrate consumption from both in-vivo and in-vitro studies. Since numerous metabolic activity of the bacteria associated with the oral disease development results symptoms of low $\mathrm{pH}$, the new invention in this study potentially an alternative approach in prevention and treatment of periodontal diseases, caries and dental erosion.

Keywords: $\mathrm{pH}$ rebalancer, saliva, $\mathrm{pH}$ neutralizing, mouth refreshener

Copyright (C) 2021. Korean Academy of Preventive Dentistry. All rights reserved.

This is an Open Access article distributed under the terms of the Creative Commons Attribution Non-Commercial License (http://creativecommons.org/licenses/ by-nc/4.0) which permits unrestricted non-commercial use, distribution, and reproduction in any medium, provided the original work is properly cited. 